\title{
INFLUÊNCIA DE SOLVENTES ORGÂNICOS NA ADSORÇÃO DE LINALOL E DECANAL EM SÍLICA GEL
}

\author{
Influence of organic solvents on adsorption of linalool and decanal on silica gel
}

\author{
Adriana Régia Cornélio ${ }^{1}$, Fabiana Queiroz Ferrua $^{2}$, Mário César Guerreiro ${ }^{3}$
}

\begin{abstract}
RESUMO
Estudou-se a influência de solventes orgânicos (etanol, propanol e acetato de etila) na isoterma de adsorção de uma solução-modelo do óleo essencial de laranja em sílica-gel. A solução-modelo constituiu-se de compostos oxigenados (linalol e decanal) dissolvidos em d-limoneno (solvente). A influência da temperatura no processo de adsorção foi determinada para sistemas ternário (d-limoneno + linalol + decanal) à temperatura de 298,15 K. Para o composto oxigenado decanal, os solventes que mais influenciaram no processo de adsorção foram o etanol e o acetato de etila; já para o linalol, todos os solventes estudados tiveram influência sobre o processo.
\end{abstract}

Termos para indexação: Adsorção, solventes orgânicos, linalol, decanal, d-limoneno.

\begin{abstract}
The influence of organic solvents (ethanol, propanol and ethyl acetate) on the isotherm of adsorption of model solutions of orange essential oil on silica gel was investigated. The model solution consisted of oxygenated compounds (linalool and decanal) dissolved in d-limoneno. The influence of temperature on the process of adsorption was determined by ternary systems (d-limoneno + linalool + decanal) at the temperature of $298,15 \mathrm{~K}$. For the oxygenated compound decanal, the solvents that showed greater influence on the process of adsorption were ethanol and ethyl acetate, and for linalool all of the solvents studied were shown to influence the process.
\end{abstract}

Index terms: Adsorption, organic solvents, linalool, decanal, d-limonene.

(Recebido para publicação em 7 de abril de 2003 e aprovado em 19 de agosto de 2003)

\section{INTRODUÇÃO}

O baixo consumo de suco de laranja industrializado no mercado interno está relacionado à menor aceitação desse pelo consumidor brasileiro, que rejeita o seu sabor e odor desagradável, aliada à disponibilidade do fruto "in natura" durante todo o ano, facilitando o consumo do suco fresco, cuja qualidade sensorial é considerada superior à do suco industrializado.

Durante o processamento térmico do suco de laranja, ocorre a perda de grande parte dos compostos responsáveis pelo seu sabor e aroma. Essa perda é muito elevada na produção do suco concentrado. Para melhorar a aceitação do suco concentrado, é adotado pelas indústrias o restabelecimento do seu sabor e aroma pela readição de compostos, sob a forma de óleo essencial, recuperado dos vapores liberados durante o processo de evaporação. O sabor e odor estranhos presentes no suco processado após a readição do óleo essencial é causado pela oxidação do d-limoneno, principal componente do óleo essencial de laranja. A separação e/ou concentração da fração oxigenada processo denominado de desterpe- nação é um desafio que vem sendo objeto de pesquisas realizadas mundialmente (FERRUA, 2001).

Os óleos de citrus são largamente empregados nas indústrias de alimentos, farmacêutica e de cosméticos como substâncias incrementadoras de sensações organolépticas (sabor e aroma), principalmente pelo fato de serem substâncias naturais. Os citrus representam mais de $40 \%$ do total de óleos essenciais na indústria alimentícia, sendo empregados na fabricação de sorvetes, licores, balas, caramelos, biscoitos doces, bombons, pós para refrescos, refrigerantes, além de serem incorporados nos sucos cítricos concentrados (FERRUA, 2001).

O óleo essencial de laranja obtido dos vapores na concentração do suco é constituído basicamente de uma mistura de hidrocarbonetos terpênicos, os quais são quimicamente instáveis, e compostos oxigenados, que representam 5 a $10 \%$ do óleo, e são os principais responsáveis pelo sabor e aroma característico do óleo essencial. Devido ao caráter insaturado, esses compostos oxidam-se facilmente sob a influência do ar, luz e

\footnotetext{
1. Aluna do Departamento de Ciência dos Alimentos - Universidade Federal de Lavras/UFLA - Caixa Postal 37 - 37200-000 - Lavras, MG - drilavras@yahoo.com.br

2. Professor do DCA/UFLA - fqferrua@ufla.br

3. Professor do Departamento de Química//UFLA - guerrero@ufla.br
} 
umidade. O efeito principal da oxidação desses compostos é a perda da qualidade organoléptica do óleo essencial em razão da produção de substâncias de sabores desagradáveis (ASCHERI, 1999).

Um dos problemas da reincorporação do óleo essencial ao suco de laranja concentrado, cujo objetivo inicial é melhorar sua qualidade sensorial, é a ocorrência da degradação da fração terpênica (principalmente do d-limoneno) que leva à formação de substâncias responsáveis pelo sabor indesejável, diminuindo sua aceitabilidade. Além disso, o uso da fração terpênica em produtos límpidos, tais como perfumes e bebidas, tem como inconveniente o fato de o d-limoneno ser insolúvel em solventes polares (tais como a água e álcoois), prejudicando a qualidade visual desses produtos, (FERRUA, 2001).

Diversos são os processos citados na literatura para a remoção de terpenos (processo de desterpenação) de óleos essenciais de citrus, entre eles, pode-se citar a adsorção.

A adsorção é a transferência de um ou mais constituintes (adsorbatos) de uma fase fluida (o adsorptivo) para a superfície de uma fase sólida (o adsorvente) (RUTHVEN, 1984). A retenção de compostos em uma superfície sólida pode ser devida às forças de atração existentes na superfície do sólido. O processo de separação por adsorção é baseado no fato de que alguns componentes são atraídos mais do que outros para a superfície de um dado adsorvente (RUTHVEN, 1984). Adsorventes com grupos superficiais polares preferem solutos polares, enquanto superfícies apolares preferem solutos apolares (BOHRA et al., 1994).

O fator principal que limita aplicação da técnica de separação por adsorção é o custo, geralmente associado com a etapa de recuperação dos componentes adsorvidos e da regeneração do adsorvente necessários a sua reutilização. Quando o produto de interesse são os componentes adsorvidos, é necessária sua recuperação por eluição com um solvente adequado, que, numa etapa posterior, deve ser separado dos componentes dessorvidos, permitindo sua recuperação e posterior reutilização, existindo também a necessidade de recuperação do adsorvente contaminado com o solvente (BARBOSA, 1998).

Segundo Ferrua (2001), os trabalhos que empregam o processo de adsorção para desterpenação do óleo essencial de citrus apresentam como desvantagem a baixa recuperação dos compostos oxigenados adsorvidos. Isso indica a necessidade de maiores estudos e otimização das condições de operação (temperatura, pressão, emprego ou não de um co-solvente) da etapa de adsorção e de dessorção, tendo por finalidade operar em condições nas quais a capacidade de adsorção e recuperação do material seja otimizada.

Em geral, para estudos de adsorção, são empregadas isotermas de adsorção, curvas usadas para descrever convenientemente a adsorção de solutos por sólidos a temperaturas constantes. Uma isoterma mostra a quantidade de adsorvato (soluto) adsorvida por um adsorvente, em função da concentração de equilíbrio do adsorvato (BOHN et al., 1979). Assim, nesta pesquisa, estudou-se o efeito de solventes orgânicos na adsorção de compostos oxigenados do óleo essencial de laranja na sílica gel, por meio de isotermas de adsorção.

\section{MATERIAL E MÉTODOS}

\section{Adsorvente}

Selecionou-se como adsorvente para o processo de desterpenação a sílica-gel, por causa de seu caráter hidrofílico e sua afinidade pelos compostos oxigenados. Empregou-se a sílica-gel 60 (Merck) para coluna cromatográfica, $\mathrm{pH}$ 6,5-7,5, com tamanho de partícula de 0,063 a $0,100 \mathrm{~mm}$.

A sílica-gel foi ativada para remoção de substâncias adsorvidas na sua superfície, utilizando-se estufa a vácuo Fanem (modelo 099 EV), sendo o adsorvente colocado dentro de placas de Petri, à temperatura constante de $383 \mathrm{~K}$ e pressão de $68,58 \mathrm{mmHg}$ por 16 horas, tempo a partir do qual o material não sofreu variação de massa, como citado por Ferrua (2001). Após seu resfriamento, a sílica-gel foi colocada em dessecadores por um período de 15 minutos; após esse período, o material adsorvente foi transferido para um erlenmeyer com junta esmerilhada e tampa, previamente tarado, determinando-se mediante pesagem a massa de sílica-gel ativada. O controle de umidade do material adsorvente, cujo objetivo é desativar sítios ativos da sílica-gel que possam promover reações indesejáveis, foi realizado adicionando a esse adsorvente uma quantidade de água pré-determinada (aproximadamente $4 \%$ da massa de sílica gel). A sílica-gel com teor de umidade desejado foi armazenada no erlenmeyer hermeticamente fechado dentro de dessecador a vácuo por um período mínimo 24 horas, sendo esse agitado periodicamente até o desaparecimento de todos aglomerados da sílica-gel úmida. Após o período de 24 horas, em que se considerou que o teor de umidade do material apresentava-se uniforme, o adsorvente foi dividido em frações de aproximadamente 12 gramas, sendo colocadas em pesa-filtros para posterior utilização nos experimentos. As etapas de sepa-

Ciênc. agrotec., Lavras, v. 28, n. 4, p. 863-870, jul./ago., 2004 
ração das frações e armazenamento nos pesa-filtros do adsorvente foram realizadas rapidamente, para evitar uma exposição prolongada do adsorvente às condições ambientais.

\section{Caracterização dos compostos utilizados}

Foram utilizados para a preparação das soluçõesmodelo do óleo essencial de laranja os seguintes padrões: linalol > 98,0\% (Merck), decanal 99,0\% (Sigma) e d-limoneno 94\% (Merck).

\section{Técnica analítica empregada}

A técnica adotada para quantificação foi a cromatografia em fase gasosa, empregando-se coluna capilar, em razão de sua alta eficiência de separação.

Foi empregada uma coluna capilar OV-WAX, de $30 \mathrm{~m}$ de comprimento, diâmetro interno de 0,25 mm com fase ligada de Cwax (polietilenoglicol) com espessura de $0,25 \mu \mathrm{m}$, utilizando-se um cromatógrafo Varian 3800 equipado com detector de ionização em chama (DIC), e as condições empregadas foram: seringa Hamilton de $10 \mu \mathrm{L}$ para injeção das amostras, razão de split: 1/30, volume injetado: $1 \mu \mathrm{L}$, programação de temperatura da coluna:

$\begin{array}{ccc}\text { Temperatura } & \text { Taxa de variação } & \text { Tempo } \\ 40^{\circ} \mathrm{C} & & 5 \mathrm{~min} \\ 180^{\circ} \mathrm{C} & 15^{\circ} \mathrm{C} / \mathrm{min} & 0 \\ 210^{\circ} \mathrm{C} & 35^{\circ} \mathrm{C} / \mathrm{min} & 2 \mathrm{~min}\end{array}$

Tempo total da corrida: $17,19 \mathrm{~min}$

Foi adotado o método de padrão interno (VALENTE, 1984) e como padrão utilizou-se ciclododecanona.

\section{Curva de calibração}

As soluções preparadas com concentrações conhecidas dos compostos oxigenados (decanal, linalol e $\alpha$-terpineol) foram diluídas com soluções com concentrações conhecidas do padrão interno (ciclododecanona). As amostras foram analisadas por cromatografia gasosa, num cromatógrafo Varian 3800, sendo construídas as curvas de calibração para cada um dos compostos oxigenados, relacionando a razão de área (área do composto a ser quantificado/área do padrão interno) versus a razão de concentração desses.
Na preparação das soluções para construção das curvas de calibração (Tabela 3), adotou-se o seguinte procedimento:

1) Preparou-se uma solução inicial SI para a mistura dos componentes que foram quantificados no solvente (d-limoneno), com concentração aproximadamente igual ao dobro do valor da concentração inicial desse(s) componente(s) no óleo essencial de laranja, dados obtidos por Marques (1997). Essa solução foi preparada pesando-se a(s) massa(s) do(s) componente(s) num balão volumétrico de $10 \mathrm{~mL}$ calibrado e previamente tarado utilizando-se uma balança analítica. Essa massa foi dissolvida no d-limoneno ajustando-se o volume para $10 \mathrm{~mL}$, obtendo-se a solução inicial (SI);

2) A partir da SI, foram feitas as demais soluções (S1, S2, ..., S6) por diluições sucessivas, adotando-se o método de adicionar volumes iguais utilizando uma seringa Hamilton de 100,0 $\pm 0,1 \mu \mathrm{L}$. As soluções diluídas foram preparadas a partir da diluição da solução inicial com diferentes volumes do solvente (d-limoneno). As soluções SI, S1, S2, S3, ..., S6 são denominadas de soluções de partida;

3) A cada $100 \mu \mathrm{L}$ (seringa Hamilton de $100 \mu \mathrm{L}$ ) das soluções de partida, foram adicionados $10 \mu \mathrm{L}$ (seringa Hamilton de $100 \mu \mathrm{L}$ ) de uma solução de padrão interno de concentração conhecida, obtendo-se as soluções SP1, SP2, ..., SP7, que foram empregadas para a obtenção das curvas de calibrações RA (razão de áreas) versus RC (razão de concentrações) entre o componente a ser quantificado e o padrão interno. A SI do padrão interno foi preparada de maneira similar à SI dos componentes a serem quantificados, pesando-se a quantidade apropriada do padrão interno (ciclododecanona) num balão volumétrico de $10 \mathrm{~mL}$ aferido e previamente tarado, e posteriormente diluindo-se em d-limoneno.

Determinação da concentração inicial corrigida dos componentes

Foi determinada a concentração inicial corrigida dos componentes do solvente (d-limoneno), pela análise cromatográfica, pois verificou-se a contaminação do mesmo pelos componentes (linalol e decanal). A determinação da concentração desses compostos no dlimoneno foi necessária para a correção das concentrações das soluções utilizadas nos experimentos de adsorção e conhecimento da massa real do componente que é adsorvido. A concentração inicial corrigida desses compostos no d-limoneno foi determinada empregando-se a técnica de adição de padrão. 
TABELA 1 - Procedimento para preparação das soluções da curva de calibração.

\begin{tabular}{|c|c|c|c|}
\hline $\begin{array}{l}\text { Solução } \\
\text { Partida }\end{array}$ & $\begin{array}{c}\text { Quantidade de D-limoneno (D-L) e } \\
\text { de solução Inicial (SI) }\end{array}$ & Solução SP & $\begin{array}{l}\text { Quantidade de Solução Partida e de } \\
\text { Solução Padrão Interno (SPI) }\end{array}$ \\
\hline S1 & $100 \mu \mathrm{L}$ D-L $+200 \mu \mathrm{L}$ SI & SP1 & $100 \mu \mathrm{L}$ SI $+10 \mu \mathrm{L}$ SPI \\
\hline S2 & $100 \mu \mathrm{L}$ D-L $+100 \mu \mathrm{L} \mathrm{SI}$ & SP2 & $100 \mu \mathrm{L} \mathrm{S} 1+10 \mu \mathrm{L}$ SPI \\
\hline S3 & $200 \mu \mathrm{L}$ D-L $+100 \mu \mathrm{L}$ SI & SP3 & $100 \mu \mathrm{L} \mathrm{S} 2+10 \mu \mathrm{L}$ SPI \\
\hline S4 & $300 \mu \mathrm{L}$ D-L + $100 \mu \mathrm{L}$ SI & SP4 & $100 \mu \mathrm{L} \mathrm{S} 3+10 \mu \mathrm{L}$ SPI \\
\hline S5 & $400 \mu \mathrm{L}$ D-L + $100 \mu \mathrm{L}$ SI & SP5 & $100 \mu \mathrm{L} \mathrm{S} 4+10 \mu \mathrm{L}$ SPI \\
\hline \multirow[t]{2}{*}{ S6 } & $500 \mu \mathrm{L}$ D-L + $100 \mu \mathrm{L}$ SI & SP6 & $100 \mu \mathrm{L} \mathrm{S} 5+10 \mu \mathrm{L}$ SPI \\
\hline & & SP7 & $100 \mu \mathrm{L} \mathrm{S6}+10 \mu \mathrm{L}$ SPI \\
\hline
\end{tabular}

$$
\mathrm{C}_{\mathrm{i}, \mathrm{SPK}}^{\mathrm{corr}} \mathrm{V}_{\mathrm{T}}=\left(\mathrm{C}_{\mathrm{i}, \mathrm{SJ}}+\mathrm{C}_{\mathrm{i}}^{0}\right) \mathrm{V}_{100}+\mathrm{C}_{\mathrm{i}}^{0} \mathrm{~V}_{10}
$$

em que:

$\mathrm{C}_{\mathrm{i}, \mathrm{SPK}}^{\text {corr }}$ : concentração corrigida da solução SPK para o componente $\mathrm{i}$;

$\mathrm{C}_{\mathrm{i}}^{0}$ : concentração contaminante do componente i no dlimoneno (solvente);

$\mathrm{C}_{\mathrm{i}, \mathrm{SJ}}$ : concentração do componente i na solução $\mathrm{SJ}$, cuja preparação está descrita no item 4 , admitindo-se que o solvente seja puro (isento de contaminantes);

$\mathrm{V}_{100}$ : volume da solução SJ empregado na preparação da solução SPK, e que corresponde a $100 \mu \mathrm{L}$;

$\mathrm{V}_{10}$ : volume da solução PI (padrão interno) empregado no preparo da solução SPK, e que corresponde a 10 $\mu \mathrm{L}$;

$\mathrm{V}_{\mathrm{T}}$ : volume total da solução SPK preparada da mistura da solução SJ e da solução do PI, e corresponde a 110 $\mu \mathrm{L}$.

No emprego dessa técnica, foram utilizados os dados obtidos na construção da curva de calibração, admitindo-se que o fator-resposta do cromatógrafo a gás (RA - razão da área do composto a ser quantificado e do padrão interno empregado) para cada composto é proporcional à sua concentração na solução injetada.

Desse modo, o fator-resposta, ou seja, a $\mathrm{RA}_{\mathrm{i}}$ (área do componente i pela área do padrão interno) será dada por:

$$
\mathrm{RA}_{\mathrm{i}}=\mathrm{K}_{\mathrm{i}} \frac{\mathrm{C}_{\mathrm{i}, \mathrm{SPK}}^{\mathrm{corr}}}{\mathrm{C}_{\mathrm{SPI}}}=\frac{\mathrm{K}_{\mathrm{i}} \mathrm{V}_{100}\left(\mathrm{C}_{\mathrm{i}, \mathrm{SJ}}+\mathrm{C}_{\mathrm{i}}^{0}\right)}{\mathrm{V}_{\mathrm{T}} \mathrm{C}_{\mathrm{SPI}}}+\frac{\mathrm{K}_{\mathrm{i}} \mathrm{V}_{10}\left(\mathrm{C}_{\mathrm{i}}^{0}\right)}{\mathrm{V}_{\mathrm{T}} \mathrm{C}_{\mathrm{SPI}}}
$$

Rearranjando a equação acima, pode-se escrever:

$$
\mathrm{RA}_{\mathrm{i}}=\mathrm{A} \frac{\mathrm{C}_{\mathrm{i}, \mathrm{SJ}}}{\mathrm{C}_{\mathrm{SPI}}}+\mathrm{B}=\mathrm{A} * \mathrm{RC}_{\mathrm{i}}+\mathrm{B}
$$

Sendo:

$$
\begin{gathered}
\mathrm{A}=\frac{\mathrm{K}_{\mathrm{i}} \mathrm{V}_{100}}{\mathrm{~V}_{\mathrm{T}}} \\
\mathrm{B}=\frac{\mathrm{K}_{\mathrm{i}} \mathrm{C}_{\mathrm{i}}^{0}}{\mathrm{C}_{\mathrm{SPI}}}
\end{gathered}
$$

em que:

$\mathrm{C}_{\mathrm{SPI}}$ : concentração da solução de padrão interno empregada nas soluções;

$\mathrm{RC}_{\mathrm{i}}$ : razão de concentração do composto i na solução SP pela concentração do componente i na solução do padrão interno.

Os valores de A e B são determinados por uma regressão linear por mínimos quadrados dos dados experimentais $\mathrm{RA}_{\mathrm{i}}$ versus $\mathrm{RC}_{\mathrm{i}}$, e a concentração inicial do componente i pode ser obtida pela relação:

$$
\mathrm{C}_{\mathrm{i}}^{0}=\frac{\mathrm{BC}_{\mathrm{SPI}} \mathrm{V}_{10}}{\mathrm{AV}_{\mathrm{T}}}
$$

Determinando-se a concentração contaminante do componente i no $d$-limoneno, essa foi empregada para corrigir as concentrações das soluções preparadas, conseguindo-se, dessa forma, as verdadeiras concentrações de cada componente. Para isso, foi empregada a equação (1.6).

\section{Estudo da adsorção em sistema em batelada}

Os experimentos de adsorção dos compostos oxigenados na sílica-gel para obtenção da isoterma de 
adsorção foram realizados em sistema em batelada agitado. A sílica-gel com teor de umidade controlado foi pesada numa balança analítica dentro da célula de adsorção ( $\pm 1 \mathrm{~g}$ ), de massa previamente conhecida. Foram empregados como célula de adsorção frascos erlenmeyers de 25 $\mathrm{mL}$ com tampa esmerilhada. Após a pesagem, foi inserido o solvente $(5 \mathrm{~mL})$ por meio de uma seringa de vidro hipodérmica; em seguida, a célula foi imediatamente fechada com a tampa de vidro esmerilhada e colocada num dessecador. A quantidade de adsorbato e solvente adicionado na célula de adsorção foi determinada por diferença de massa. Pesou-se, em um recipiente previamente tarado, a quantidade de adsorbato e solvente desejados, transferindo-a para a célula de adsorção. A célula de adsorção, após a adição da mistura, era imediatamente fechada e colocada sob agitação à temperatura controlada. $\mathrm{O}$ sistema empregado para agitação e controle de temperatura foi uma incubadora refrigerada Shaker (agitador) Modelo: MA 830/A, marca: Marconi, que permite a realização de até 24 experimentos num mesmo intervalo de tempo. Então, o sistema mistura sintética do óleo essencial/adsorvente permanece sob contato por um tempo 24 horas para atingir o equilíbrio prático. Para cada tratamento, foi retirada uma amostra da solução líquida com seringa hipodérmica de vidro (5 $\mathrm{mL}$ ), que posteriormente foi filtrada em unidade filtrante Millex $13 \mathrm{~mm}$ em polietileno com membrana PTFE (politetrafluoretileno), $0,22 \mu \mathrm{m}$ de poro (Millipore) para separação das partículas do adsorvente da fase fluida. Essas amostras filtradas foram armazenadas em freezer $(\mathrm{T}=255,15 \mathrm{~K})$ em frascos de vidro com rosca cobertos com filme de PVC antes de serem fechados, até serem realizadas as análises cromatográficas.

A importância do emprego de solventes para o processo de adsorção é a eluição dos compostos que foram adsorvidos pela sílica gel. O estudo do processo de dessorção é importante quando os compostos de maior interesse ficam retidos no adsorvente, podendo os solventes facilitarem o processo.

\section{Determinação da concentração do composto oxigenado na fase fluida e adsorvida}

As concentrações para os componentes na fase fluida são determinadas experimentalmente por meio de análises cromatográficas da fase fluida. A determinação da concentração do componente i no equilíbrio na fase fluida é dado por:

$$
\mathrm{C}_{\mathrm{if}}^{\mathrm{eq}}=\frac{\mathrm{C}_{\mathrm{i}} \mathrm{V}_{\mathrm{T}}}{\mathrm{V}_{100}}
$$

em que:
$\mathrm{C}^{\mathrm{eq}}$ if: concentração inicial do componente i no equilíbrio na fase fluida $\left[\mathrm{g} / \mathrm{m}^{3}\right]$

$\mathrm{C}_{\mathrm{i}}$ : concentração componente $\mathrm{i}$ obtida da curva de calibração corrigida do componente $i\left[\mathrm{~g} / \mathrm{m}^{3}\right]$

$\mathrm{V}_{\mathrm{T}}$ :volume total da fase fluida, correspondente a 110 $[\mu \mathrm{L}]$

$\mathrm{V}_{100}$ : volume da fase fluida, correspondente a $100[\mu \mathrm{L}]$ A concentração inicial da fase fluida foi calculada por:

$$
\mathrm{C}_{\mathrm{if}}^{0}=\frac{\mathrm{m}_{\mathrm{it}}}{\mathrm{V}_{\mathrm{i}}}
$$

em que:

$\mathrm{m}_{\mathrm{it}}$ : massa total do componente $\mathrm{i}$ na fase fluida $[\mathrm{g}$ ]

$\mathrm{V}_{\mathrm{i}}$ : volume de solvente utilizado no preparo da fase fluida $\left[\mathrm{m}^{3}\right]$

Então, pelo balanço de massa, a quantidade do componente i na fase adsorvida é dada por:

$$
q=\frac{\left(C_{\text {if }}^{0}-C_{\text {if }}^{\text {eq }}\right) V_{i}}{m_{\text {ads }}}
$$

em que:

$\mathrm{m}_{\mathrm{ads}}$ : massa de adsorvente empregado no experimento [g]

q: quantidade do componente i adsorvido [g do componente $\mathrm{i} / \mathrm{g}$ do adsorvente]

\section{RESULTADOS E DISCUSSÃO}

\section{Adsorção para sistemas ternário}

\section{Sem adição de solvente}

Foi estudado o efeito do solvente (etanol, acetato de etila e propanol) no processo de dessorção do sistema ternário (linalol + decanal + d-limoneno) à temperatura de 298,15 K. Foram realizadas três injeções de cada amostra, sendo a amostra preparada uma única vez.

Na Figura 1 verifica-se a isoterma para a substância linalol, no sistema ternário (linalol + decanal + dlimoneno) e no sistema binário (linalol + d-limoneno) sem o uso de solventes.

É possível observar uma melhor adsorção para o linalol no sistema binário, mostrando que, 
provavelmente, há uma competição com o decanal, presente no sistema ternário, pelos sítios de adsorção.

Na Figura 2 apresenta-se a isoterma para o decanal, no sistema ternário (linalol + decanal + dlimoneno) e no sistema binário (decanal + d-limoneno) sem uso de solventes.

É possível observar que não houve diferença no processo de adsorção para o decanal nos dois sistemas, em razão provavelmente de uma maior afinidade pela sílica gel, quando comparado ao

\section{Linalol}

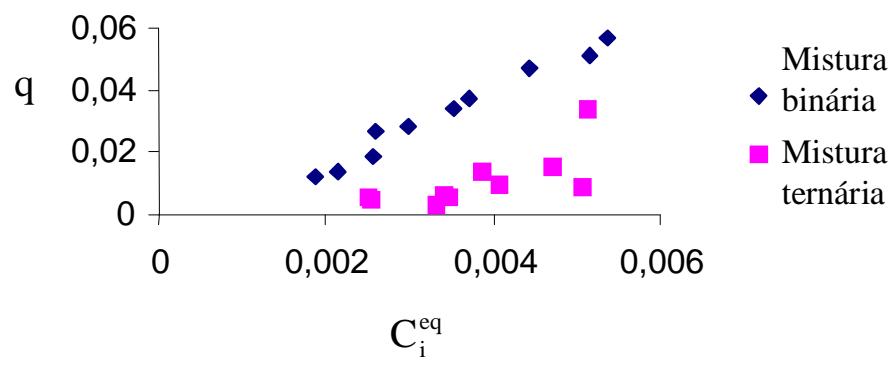

FIGURA 1 - Isoterma para o linalol no sistema ternário e no binário, a 298,15 K.

\section{Decanal}

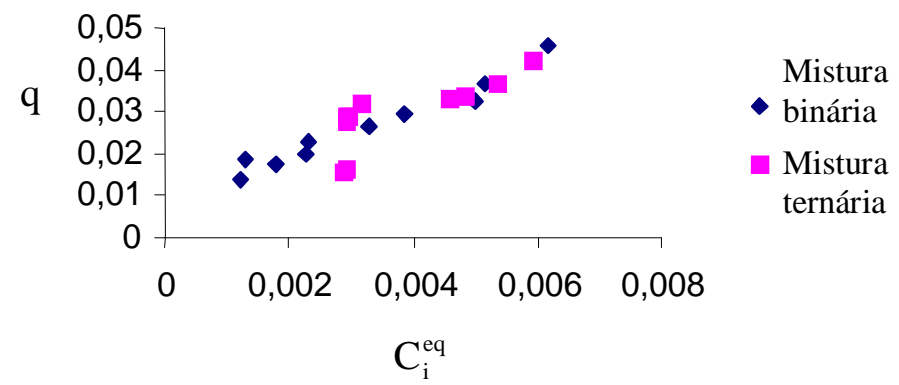

FIGURA 2 - Isoterma para o decanal no sistema ternário e no binário, a 298,15 K.

Efeito Solvente - Linalol

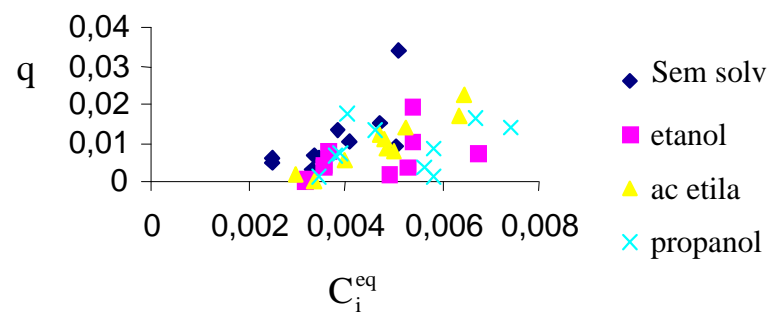

FIGURA 3 - Comportamento da isoterma de adsorção para uso de solventes para o linalol na mistura ternária (dlimoneno + linalol + decanal) a 298,15 K. 
O comportamento da isoterma de adsorção para a substância linalol (Figura 3) empregando solventes para promover o processo de dessorção foi influenciado por todos os solventes empregados.

O comportamento das isotermas de adsorção para a substância decanal (Figura 4) evidencia que o propanol foi o solvente que mais influenciou no processo de dessorção do composto.

Sobre o emprego do etanol e do acetato de etila, possivelmente são inadequados para serem utilizados como cossolventes num processo de dessorção devido ao efeito sinérgico com o decanal.

Esses resultados discordam de Marques (1997), que verificou durante a adsorção do óleo es- sencial de laranja numa coluna de sílica gel o deslocamento do decanal e outros compostos oxigenados pelo etanol, sendo a curva de ruptura do decanal a primeira a eluir pela coluna. A contradição desses resultados talvez seja explicada pela alta concentração dos compostos utilizados no presente trabalho ou por diferentes teores de umidade no adsorvente utilizado.

Na Figura 5 verifica-se o cromatograma da mistura ternária (d-limoneno + linalol + decanal) com o padrão interno (ciclododecanona) utilizado. Aparece também no cromatograma o contaminante $\alpha$-terpineol, um composto oxigenado que estava presente no dlimoneno.

\section{Efeito solvente - Decanal}

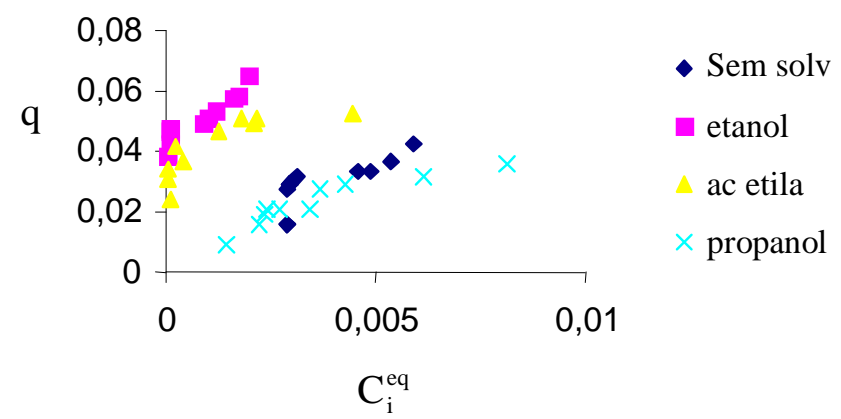

FIGURA 4 - Comportamento da isoterma de adsorção para uso de solventes para a substância decanal na mistura ternária (d-limoneno + linalol + decanal) à temperatura de 298,15 K.

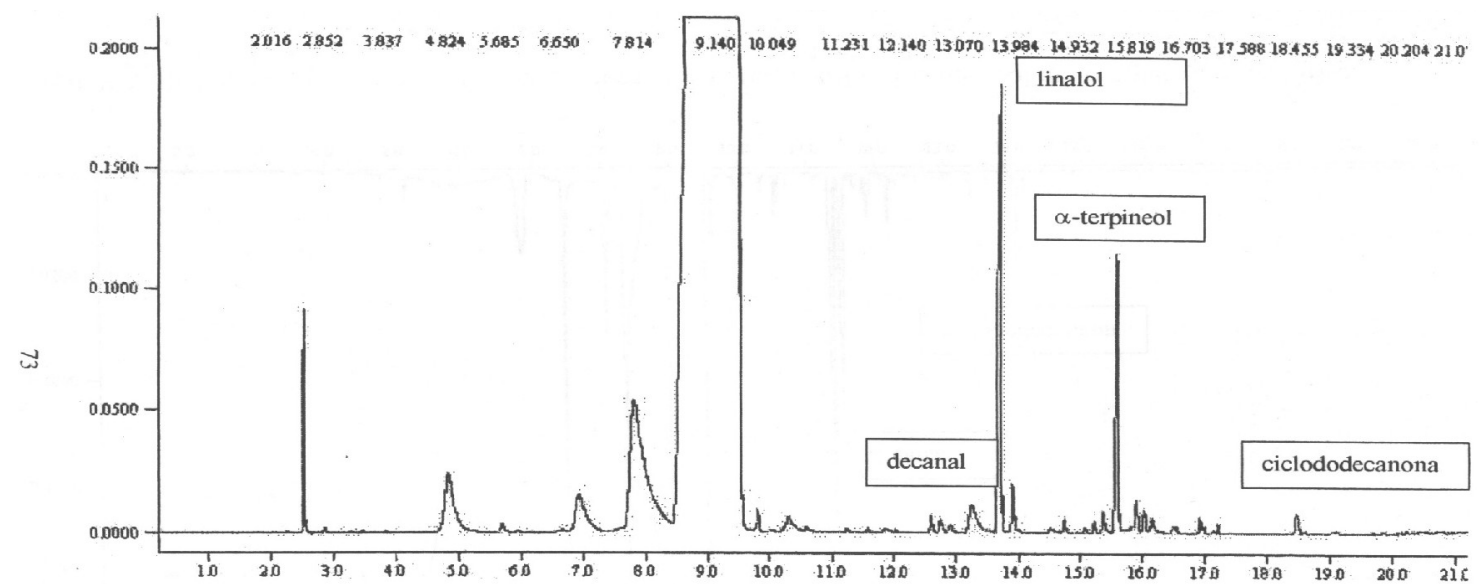

FIGURA 5 - Cromatograma dos compostos oxigenados linalol e decanal utilizando coluna capilar (OV-WAX), detector de ionização de chama e padrão interno (ciclododecanona). 


\section{CONCLUSÕES}

a) Para o sistema ternário (decanal + linalol + dlimoneno), o decanal apresentou uma maior afinidade que o linalol pela sílica gel.

b) O emprego do etanol e do acetato de etila provocou um efeito sinérgico na adsorção do decanal.

c) A isoterma de adsorção do linalol foi influenciada por todos os solventes empregados.

\section{REFERÊNCIAS BIBLIOGRÁFICAS}

ASCHERI, D. P. R. Estudo das características de adsorção de água e da estabilidade das microcápsulas de óleo essencial laranja na seleção de material de parede. 1999. Disponível em: Ǩhttp://www.scielo.com br./ciênciaetecnologiadealimentos.htm>. Acesso em: 21 nov. 2002.

BARBOSA, M. Estudo cinético de adsorsão, modelagem dinâmica e otimização de processo contínuo de purificação de cefalosporina C. 1998. 167 f. Tese (Doutorado em Engenharia de Alimentos) - Faculdade de Engenharia de Alimentos, Universidade Estadual de Campinas, Campinas, 1998.

BOHN, H. L.; McNEAL, B. L.; O'CONNOR, G. A. Soil chemistry. New York: John Wiley \& Sons, 1979. 329 p.
BOHRA, P. M.; VAZE, A. S.; PANGALAR, V. G.; TASKAR, A. Adsorptive recovery of water soluble essential oil components. Journal Chemical Technology Biotechnology, [S.1.], v. 60, p. 97-102, 1994.

FERRUA, F. Q. Estudo do processo de desterpenação do óleo essencial de laranja por adsorção: seleção de adsorventes e modelagem do processo. 2001. $427 \mathrm{f}$. Tese (Doutorado em Engenharia de Alimentos) - Faculdade de Engenharia de Alimentos, Universidade Estadual de Campinas, Campinas, 2001.

MARQUES, D. S. Desterpenação do óleo essencial de laranja por cromatografia preparativa de fluido supercrítico. 1997. Dissertação (Mestrado em Engenharia de Alimentos) - Faculdade de Engenharia de Alimentos, Universidade Estadual de Campinas, Campinas, 1997.

RUTHVEN, D. M. Principles of adsorption and adsorption process. New York: Wiley, 1984.

VALENTE, A. L. P. Desenvolvimento e avaliação de métodos cromatográficos para compostos do tipo $\mathbf{C}_{\mathbf{x}} \mathbf{C l}_{\mathbf{y}} \mathbf{B r}_{\mathbf{z}} \mathbf{H}_{\mathrm{N}}$. 1984. 20 f. Tese (Doutorado em Química) - Instituto de Química, Universidade Estadual de Campinas, Campinas, 1984. 